\title{
Macro Creatine Kinase: Comparison of Different Screening Methods and Revision of the Bibliography
}

\section{Pérez de Ciriza C and Nerea Varo*}

Clinical Chemistry Department, Clínica Universidad de Navarra, Pamplona, Spain

*Corresponding author: Nerea Varo, Servicio de Bioquímica, Clínica Universidad de Navarra, Avda Pío XII 36, 31008 , Pamplona, Spain, Tel: +34 948 296 395; Fax: +34 948296 500; E-mail: nvaro@unav.es

Received date: 10 Nov, 2014; Accepted date: 12 Nov, 2014; Published date: 14 Nov, 2014

Copyright: (c) 2014 Pérez de Ciriza C et al. This is an open-access article distributed under the terms of the Creative Commons Attribution License, which permits unrestricted use, distribution, and reproduction in any medium, provided the original author and source are credited.

\begin{abstract}
Introduction: Macro-creatine kinase (Macro-CK) is a complex with longer half-life that leads to elevation of enzyme activity and analytical errors. We present two cases with macro-CK to picture the problem.

The aim of the study was to evaluate and compare different methods using polyethylene glycol (PEG) for screening.

Methods: Serum samples $(n=39)$ were analysed using different PEG methods PEG6000, 30 minutes centrifugation, $3000 \mathrm{rpm}, \mathrm{PEG} 8000,10$ minutes incubation and 5 minutes centrifugation, $1000 \mathrm{~g}$ Modified method 1, 10 minutes centrifugation. The recovery percentage and the polyethylene glycol precipitation activity (PPA) were calculated. In four patients macro-CK was confirmed by electrophoresis (Sebia).

Results: The recovery percentage was significantly higher in all non-macro-CK samples than in macro-CK $(p<0.001$ ) (Method 1: $80.6 \pm 7.9 \%$; (2): $60.2 \pm 10.4 \%$ and (3): $79.9 \pm 8.7 \%$ vs. Method 1: $13.8 \pm 5.0 \%$; (2): $12.4 \pm$ $3.2 \%$ and (3): $8.7 \pm 9.0 \%$ ). No differences in recovery percentages were found between normal or elevated CK values. No differences were observed between methods 1 and $3(p=0.453)$ and both were concordant (Confidence interval $95 \%$ was $-8.819,9.153)$. However, significant differences were observed between method 2 and the others $(p<0.0001)$
\end{abstract}

Conclusion: Method 3 is an adequate method for screening which would lead to a better identification of macroCK interferences.

Keywords: Macroenzymes; Electrophoresis; Polyethylene glycol; Creatine kinase; Precipitation

\section{Abbreviations: \\ ADP: Adenosine Diphosphate; ATP: Adenosine Triphosphate; CK: Creatine Kinase; Macro-CK: Macro Creatine Kinase; PBS: Phosphate Buffered Saline; PEG: Polyethylene Glycol; PPA: Polyethylene Glycol Precipitation Activity}

\section{Introduction}

Macroenzymes are high-molecular weight complexes generated by the polimerization of normal enzymes and immunoglobulins or other molecules such as lipoproteins, proteins, cell membrane fragments, drugs or the same molecule (autopolimerization).

Macroenzymes have longer half-life in systemic circulation and can normally be found in serum under physiologic or pathophysiologic conditions (hypothyroidism, malignancies, autoimmune diseases among others) [1].

Macroenzymes are responsible of elevated enzyme activity leading to erroneous interpretation and causing diagnostic confusion [2] Also, inadequate understanding of macroenzymes lead to increased the number of unnecessary repetitions and procedures delaying the diagnosis and raising the costs.

Creatine kinase (CK) is an enzyme that catalyzes the conversion of creatine to phosphocreatine, consuming adenosine triphosphate (ATP) and generating adenosine diphosphate (ADP). It is a dimeric enzyme that forms 3 different isoenzymes: CK-BB, CK-MM and CK$\mathrm{MB}$ by the pairing of $\mathrm{M}$ and $\mathrm{B}$ subunits. $\mathrm{CK}$ isoenzyme $\mathrm{MB}$ is usually analysed along with total $\mathrm{CK}$ in patients with suspicion of myocardial infarction. CK-MB rises around 4-6 hours after the event, peaks within 12-24 hours, and returns to baseline levels within 24-48 hours.

Macro-CK is present in around $2.6 \%$ of patient samples and it is generally associated with moderate elevation of total CK activity [2] resulting in contradictory results between clinical parameters and laboratory results and increasing the tests and procedures performed and the time to confirm the results. Thus, it is crucial to detect and confirm the presence of macro-CK. Polyethylene glycol precipitation (PEG) methods are described for screening, however, there is not still a well established and validated method [3]. The aim of the present work is to compare different PEG methods described in the literature and asses the most adequate screening method, as well as revising the bibliography regarding macro-CK. 


\section{Materials and Methods}

Serum samples from 39 patients were obtained in Vacutainer tubes. Total CK activity was measured in a Roche Modular Analytics P analyser (Roche) by the Szasz method as well as CK-MB isoform in a DxC 800 (Beckman Coulter) using antibodies inhibiting CK-M subunit. PEG is known to retract solvent molecules from immunoglobulins, proteins and lipids, resulting in an increased protein concentration and finally precipitation [4]. Three different polyethylene glycol (PEG) precipitation methods were performed.

The first protocol (Method 1, PEG6000) is the method routinely performed in our laboratory for detection of macroprolactinemia. One hundred $\mu \mathrm{L}$ of serum were mixed with an equal volume of PEG 6000 (Merck, Art. 807491) $250 \mathrm{~g} / \mathrm{L}$ in PBS-buffer (Phosphate Buffered Saline, Sigma Art. P4417) and vortex mixed for 1 min. Next, centrifugation was performed at $3000 \mathrm{rpm}$ for $30 \mathrm{~min}$ resulting in a clear supernatant with precipitate at the bottom. Simultaneously, a dilution of the serum was prepared and enzyme activities were measured both on the supernatant and the dilution.

The second protocol (Method 2, PEG8000) [5] was performed with one hundred $\mu \mathrm{L}$ of PEG 8000 (Sigma, Art. P-5413) $250 \mathrm{~g} / \mathrm{L}$ in PBSbuffer and one hundred $\mu \mathrm{L}$ of serum and vortex mixed for 30 seconds followed by 10 minutes incubation at room temperature. Afterwards a shorter but more vigorous centrifugation step was performed (5 minutes, $10000 \mathrm{~g}$ ). Serum samples were diluted and CK was measured in both the dilution and the supernatant.

The third protocol (Method 3) consisted of a modification of method 1, shortening the long and time-consuming centrifugation step to 10 minutes. Besides, repeatability of this method was analyzed in 15 samples due to its similarity to method 1 (adequate concordance) and because of being less time-consuming.

The recovery percentage and the polyethylene glycol precipitation activity (PPA) percentage were calculated for all methods using the following formulas:

Recovery (\%) = CK-MB activity PEG/ CK-MB activity PBS x 100

PPA $(\%)=[($ CK-MB activity PBS - CK-MB activity PEG) / CK-MB activity PBS] x 100

Four macro-CK cases were confirmed by electrophoresis in an Hydrasys analyser (Sebia) using a semi-automated agarose gel electrophoresis system and the Hydrasys Hydragel ISO-CK assay (Sebia), according to the manufacturer instructions. Samples were run simultaneously with a control sample.

\section{Statistical Analysis}

Statistical analyses were performed using the SPSS 15.0 package (SPSS, Chicago, IL) and STATA 12.0. Normality of continuous variables was assessed using Shapiro-Wilks test. Comparisons of continuous variables between two groups were performed by T-test. Bivariant correlations were studied by Pearson test. Related samples were studied with the T-test for related samples. Method comparison was performed with the Bland-Altman test. Expected values for recovery percentage as well as PPA in all methods were calculated as mean \pm 2 S.D. To study the repeatability the mean of the duplicates and the absolute value differences were calculated. The limits of acceptability for the repeatability study corresponded to the mean differences multiplied by 4 . All results are expressed as mean and standard deviation (S.D.). $\quad \mathrm{P}<0.05$ was considered statistically significant.

\section{Results}

\section{PEG recovery and PPA}

Samples in which the presence of macro-CK was suspected $(n=4)$ were analyzed along with 35 controls using different PEG precipitation methods.

The recovery was higher and the PPA percentage was significantly lower in controls than in patients for all methods $(\mathrm{p}<0.0001)$ (Table 1 and Figure 1).

\begin{tabular}{|l|l|l|l|l|l|}
\hline \multicolumn{4}{|l|}{ NON macro-CK } & \multicolumn{3}{l|}{ Macro-CK } & \\
\hline Method & Recovery (\%) & PPA (\%) & Recovery (\%) & PPA (\%) & p value \\
\hline 1 & $80.6 \pm 7.9$ & $19.4 \pm 7.9$ & $13.8 \pm 5.0$ & $\begin{array}{l}86.5 \\
5.0\end{array}$ & $p<0.0001$ \\
\hline 2 & $60.2 \pm 10.4$ & $39.8 \pm 10.4$ & $12.4 \pm 3.2$ & $\begin{array}{l}89.8 \\
3.2\end{array}$ & $p<0.0001$ \\
\hline 3 & $79.9 \pm 8.7$ & $20.1 \pm 8.7$ & $8.7 \pm 9.0$ & $\begin{array}{l}84.7 \\
9.0\end{array}$ & $p<0.0001$ \\
\hline
\end{tabular}

Table 1: Recovery percentages (\%) and PPA percentages (\%) in samples with and without macro-CK

Method 1 was more time-consuming because of the 30 minutes centrifugation step. The same 35 samples from non macro-CK, including normal and elevated CK activity, were analyzed centrifuging for 10 minutes instead of 30 . There were no statistically significant differences ( $\mathrm{p}=0.453$ ) when the centrifugation was reduced ( 30 vs 10 minutes PEG6000: Recovery $80.6 \pm 7.9 \%$ vs $79.9 \pm 8.7 \%$; PPA $19.4 \pm$ $8.0 \%$ vs $20.1 \pm 8.7 \%)$. Correlation between both methods was highly significant $(\mathrm{r}=0.957, \mathrm{p}<0.0001)$

Figure

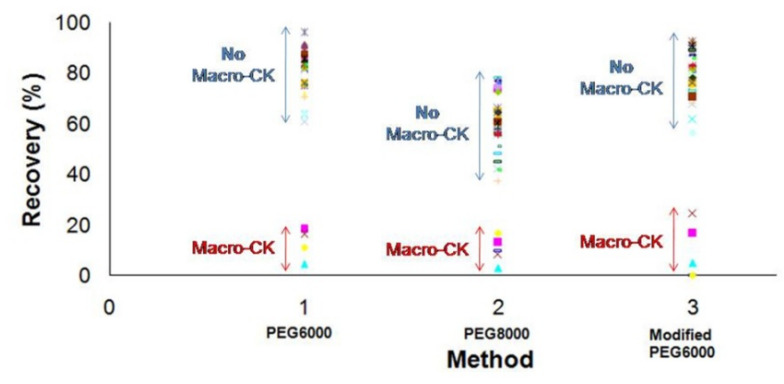

Figure 1: Individual data plots show recovery percentages (\%) in macro-CK $(n=4)$ and non macro-CK patients $(n=35)$ in the different analytical methods (Method 1,2 and 3) 
No differences were observed between normal $(n=17)$ or elevated CK values $(\mathrm{n}=18)$ in the control samples by any method (Method 1 PEG6000, $\mathrm{p}=0.709$; Method 2-PEG8000, $\mathrm{p}=0.720$; Method 3, $\mathrm{p}=0.792$ ) (Figure 2).

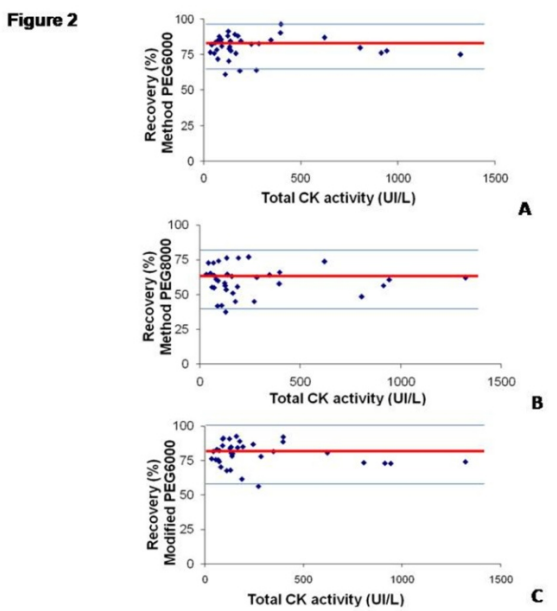

Figure 2: Graphs show no differences in recovery percentage according to total CK activity (UI/L). The recovery percentage (\%) from different methods versus total CK activity (UI/L). (A) Method 1 PEG6000, (B) Method 2 PEG8000, (C) Method 3 modified PEG6000. Mean \pm 2 SD is represented by lines.

Concordance was studied using the Bland-Altman method. Method 1 (PEG6000) and method 3 (10-minute centrifugation) were concordant but not method 2 (PEG8000 method). The confidence interval $95 \%$ for the concordance were $-8.819,9.153$. Non-significant intervals mean concordance.

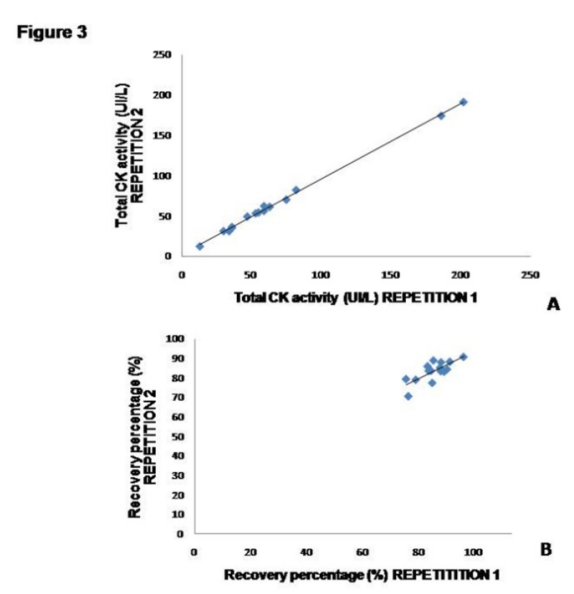

Figure 3: Repeatability study for method 3 show concordance between repetitions. Repetition 1 is represented versus repetition 2: (A) Total CK activity and (B) Recovery percentage (\%)

Repeatability ( $\mathrm{n}=15)$ was studied for method 3 (PEG6000, 10 minute-centrifugation) due to its adequate concordance to method 1 and because of being less time-consuming. The mean of the duplicates and the absolute value differences were calculated. The limits of acceptability for the repeatability study corresponded to the mean differences multiplied by 4 (Acceptability value 13.7, minimum value 0 , maximum value 6 for the recovery percentage). None of the duplicates overcame those limits. High concordance was observed between methods 1 and $3(y=0.932 x+2.462, R$ square value 0.998$)$ (Figure 3).

Confirmation of macro-CK samples in which the recovery percentage lead to a macro-CK suspicion was performed by electrophoresis. Figure 1 shows the electrophoresis patters of macroCK type 1 (Figure $1 \mathrm{~A}$ ) and type 2 (Figure $1 \mathrm{~B}$ ) by electrophoresis in agarose gel of two representative samples.

\section{Expected values for each method}

Normal ranges were determined for both methods using control samples. The recovery and PPA percentages were calculated. Normal ranges were calculated as mean $\pm 2 \mathrm{SD}$ and are shown in Table 2 .

\begin{tabular}{|l|l|l|l|l|}
\hline & \multicolumn{2}{|l|}{ NON macro-CK } & \multicolumn{2}{l|}{ Macro-CK } \\
\hline Method & Recovery (\%) & PPA (\%) & Recovery (\%) & PPA (\%) \\
\hline 1 & $64.8-96.4$ & $3.6-35.2$ & $0-25.8$ & $74.1-100$ \\
\hline 2 & $39.4-81.0$ & $19.0-60.6$ & $0-22.3$ & $77.7-100$ \\
\hline 3 & $62.5-97.4$ & $2.6-37.5$ & $0-27.7$ & $72.2-100$ \\
\hline
\end{tabular}

Table 2: Expected values for recovery and PPA percentages (\%) in non macro-CK and macro-CK patients

\section{Discussion}

Macroenzymes present higher molecular mass than the corresponding enzymes causing falsely increased total serum enzyme levels leading to unnecessary and often invasive additional diagnostic procedures.

Macroenzymes can be found in apparently healthy individuals or associated with certain diseases such as autoimmune diseases or malignant lesions and are more frequently associated with advanced age [6]. According to the literature the same patient hardly ever presents different macro-enzymes. There are few cases reported: association of macro-CK and macro-LDH in a girl affected by ulcerative colitis [7], a 68-year-old female patient presenting with increased macroamylase and macro-creatine [8] and simultaneous presence of macroamylasemia and macrolipasemia in a patient with gluten enteropathy (celiac disease) [9].

Macro-CK is present in around $2.6 \%$ of patient samples and it is generally associated with moderate elevation of total CK activity [2]. The presence of macro-CK can lead to increased CK values as well as high index (CK-MB/CK ratio), however normal CK values cannot rule out the presence of macro-CK (as in case 2). Macro-CK should be considered in patients with CK-MB concentrations exceeding $50 \%$ of total enzyme activity, because values greater than $30 \%$ are rarely found even in patients with myocardial infarction. Clinically, the absence of symptoms or an isolated and persistently increased CK favor the presence of macro-CK.

The elevated CK values and index are the consequence of analytical interferences. In the quantification method the inhibition of all $\mathrm{M}$ 
Page 4 of 5

subunits by anti-M antibody allows determination of residual $B$ subunit enzyme activity. Increased amount of CK-BBimmunoglobulin complex (macro-CK type 1) is resistant to inhibition by the anti-M antibody. On the other hand, macro-CK type 2 is not structurally related to the $\mathrm{M}$ or $\mathrm{B}$ subunits, nor is it inhibited by monoclonal anti-M antibody. So, both macro-CK types 1 and 2 can cause a falsely elevated CK-MB activity.

Biochemically macroenzymes in general and macro-CK in particular can be classified into two groups (type 1 and type 2) according to their electrophoretic characteristics and immunologic properties (Figure 4).

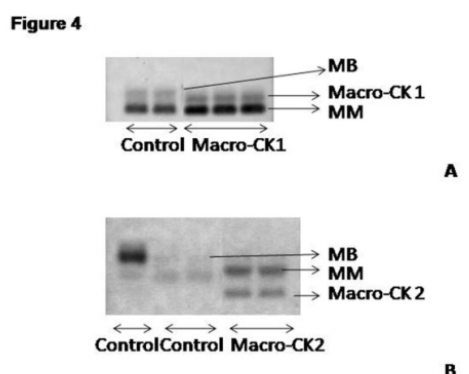

Figure 4: Picture shows representative migration patterns of $\mathrm{CK}$ isoforms and macroenzymes by gel electrophoresis. (A) Control with elevated CK activity and MB isoform and Macro-CK type 1, (B) Control 1 with elevated CKMB activity, control 2 with normal $\mathrm{CK}$ activity and $\mathrm{MB}$ isoform and Macro-CK type 2.

Macro-CK type 1 is typically characterized by a CK-BB isoenzyme and IgG complex although complexes are less frequently formed with $\operatorname{IgA}$ or IgM. The prevalence of macro-CK 1 has been reported to range from $0.54 \%$ to $2.3 \%$ [10]. However, its prevalence depends on the method of determination, age, sex and disease characteristics being more common among women [11] and in patients above 70 years old [12]. In the electrophoresis, macro-CK 1 is localized in-between CK$\mathrm{MM}$ and CK-MB more cathodically in comparison with CK-MM. Macro-CK 1 has been associated with different disorders including hypothyroidism, malignancies, autoimmune diseases, gastrointestinal and cardiovascular disease $[1,13,14]$.

Macro-CK type 2 is typically considered to originate from mitochondria (oligomeric mitochondrial $\mathrm{CK}$ ) and it is released to circulation after the mitochondrial membrane rupture during tissue disruption. The prevalence of macro-CK 2 has been reported to range from $0.5-3.7 \%$ [15]. In the electrophoresis, macro-CK 2 is localized more cathodically in comparison with CK-MM. Macro-CK 2 is more commonly associated with patients with malignancies [16], liver disease and those who are critically ill or who have widespread tissue damage $[2,17]$. Its occurrence has also been associated with a higher mortality rate $[2,15]$ and it has been even proposed as a diagnostic marker in patients with colorectal cancer [6].
The electrophoresis is the current method used in our clinical laboratory to confirm the presence of macro-CK in a sample and it is the method of choice for many clinical laboratories. PEG precipitation is a simple and effective additional test described in the literature for the detection of macroenzymes when the plasma enzyme activity is elevated [3] and can be used as a screening method reducing time, laboratory resources and costs.

However, in the literature, different PEG precipitation methods are described and sometimes the information provided is scarce. Furthermore, caution should be taken when analyzing samples and the results need to be compared with reference ranges determined by PEG precipitation in normal subjects [4].

Different studies suggested possible reference recovery intervals using PEG precipitation methods. Davidson et al. [3] reported an interval of $63-88 \%$ and Wyness et al. [18] proposed $36-85 \%$.

In our study, the results were similar to those published however; the novelty of our study is the comparison between different PEG methods. 35 non macro-CK patients were studied and different recovery intervals are proposed (Method $165-96 \%$, Method $239-81 \%$, Method 3 62-97\%). Besides, we confirmed that recovery is independent of CK activity. Different recovery percentages were observed for the macro-CK patients (Method $10-26 \%$, Method 2 $0-22 \%$, Method $30-28 \%$ ). The differences in recovery percentage between macro-CK and non macro-CK samples made it possible to fully distinguish both categories. However, electrophoresis should be used in non-conclusive samples. All methods can be used for screening because of the different recovery percentages between macro-CK and non macro-CK patients. Due to the better results shown by methods 1 and 3, the use of those methods is suggested. Besides, the reproducibility of method 3 as well as the reduced time results in an adequate, rapid and reproducible screening method.

\section{Conclusion}

In conclusion, the role of the laboratory in detection of macro-CK can be crucial to establish an adequate follow-up methodology for the patient and to reduce unnecessary costs.

Detection of macro-CK can be screened using the PEG precipitation method and confirmed in the laboratory by electrophoresis. Precipitation using PEG is an adequate method for the screening of macro-CK. The best method would be method 3 because of its rapidity and reproducibility. This method would allow the identification of interferences caused by macro-CK, considerably reducing expenses and misinterpretation of the laboratory data.

\section{References}

1. Hsiao JF, Ning HC, Gu PW, Lin WY, Chu PH (2008) Clinical role of recurrently elevated macro creatine kinase type 1. J Clin Lab Anal 22: 186-191.

2. Lee KN, Csako G, Bernhardt P, Elin RJ (1994) Relevance of macro creatine kinase type 1 and type 2 isoenzymes to laboratory and clinical data. Clin Chem 40: 1278-1283.

3. Davidson DF, Watson DJ (2003) Macroenzyme detection by polyethylene glycol precipitation. Ann Clin Biochem 40: 514-520.

4. Fahie-Wilson M, Halsall D (2008) Polyethylene glycol precipitation: proceed with care. Ann Clin Biochem 45: 233-235.

5. Patteet L, Simoens M, Piqueur M, Wauters A (2012) Laboratory detection of macro-aspartate aminotransferase: case report and evaluation of the PEG-precipitation method. Clin Biochem 45: 691-693. 
Citation: Pérez de Ciriza C, Varo N (2014) Macro Creatine Kinase: Comparison of Different Screening Methods and Revision of the Bibliography. Biochem Anal Biochem 3: 161. doi:10.4172/2161-1009.1000161

Page 5 of 5

6. Galasso PJ, Litin SC, O'Brien JF (1993) The macroenzymes: a clinical review. Mayo Clin Proc 68: 349-354.

7. Pascarella F, Caropreso M, Miele E, Fortunato G, Vajro P, et al. (2007) Macro-creatine kinase and macro-lactate dehydrogenase in a girl with ulcerative colitis. Dig Liver Dis 39: 780-781.

8. Gallucci F, Madrid E, Esposito P, Uomo G (2007) Association of macroamylasemia and type I macro-creatine kinasemia. A case report. JOP 8: 605-608.

9. Zaman Z, Van Orshoven A, Mariën G, Fevery J, Blanckaert N (1994) Simultaneous macroamylasemia and macrolipasemia. Clin Chem 40: 939-942.

10. Sturk A, Sanders GT (1990) Macro enzymes: prevalence, composition, detection and clinical relevance. J Clin Chem Clin Biochem 28: 65-81.

11. Davidson DF, Scott JG (2012) Detection of creatine kinase macroenzymes. Ann Clin Biochem 49: 482-485.

12. Etienne E, Hanser AM, Woehl-Kremer B, Mohseni-Zadeh M, Blaison G, et al. (2009) [Macroenzymes: macro-ASAT and macro-CPK. Two cases and literature review]. Rev Med Interne 30: 963-969.

13. Galarraga B, Sinclair D, Fahie-Wilson MN, McCrae FC, Hull RG, et al. (2003) A rare but important cause for a raised serum creatine kinase concentration: two case reports and a literature review. Rheumatology (Oxford) 42: 186-188.

14. Laureys M, Sion JP, Slabbynck H, Steenssens L, Cobbaert C, et al. (1991) Macromolecular creatine kinase type 1: a serum marker associated with disease. Clin Chem 37: 430-434.

15. Stein W, Bohner J, Renn W, Maulbetsch R (1985) Macro creatine kinase type 2: results of a prospective study in hospitalized patients. Clin Chem 31: 1959-1964.

16. Jap TS, Wu YC, Ho DM, Chiang H (2000) Spuriously high CK-MB isoenzyme activity mimicking acute myocardial infarction in a patient with adenocarcinoma of the rectum. Zhonghua Yi Xue Za Zhi (Taipei) 63: 413-417.

17. Remaley AT, Wilding P (1989) Macroenzymes: biochemical characterization, clinical significance, and laboratory detection. Clin Chem 35: 2261-2270.

18. Wyness SP, Hunsaker JJ, La'ulu SL, Rao LV, Roberts WL (2011) Detection of macro-creatine kinase and macroamylase by polyethylene glycol precipitation and ultrafiltration methods. Clin Chim Acta 412: 2052-2057. 